\section{Histoire Épistémologie Langage}

43-2 | 2021

Linguistique psychologique et sémiotique : le contexte allemand et son influence

\title{
Linguistique psychologique et sémiotique : le contexte allemand et son influence. Introduction au dossier
}

Jean-Michel Fortis

\section{OpenEdition}

Journals

Édition électronique

URL : https://journals.openedition.org/hel/914

DOI : 10.4000/hel.914

ISSN : $1638-1580$

\section{Éditeur}

Société d'histoire et d'épistémologie des sciences du langage

Édition imprimée

Date de publication : 31 décembre 2021

Pagination : 7-30

ISBN : 9791091587150

ISSN : 0750-8069

\section{Référence électronique}

Jean-Michel Fortis, « Linguistique psychologique et sémiotique : le contexte allemand et son

influence. Introduction au dossier », Histoire Épistémologie Langage [En ligne], 43-2 | 2021, mis en ligne le 01 décembre 2021, consulté le 17 décembre 2021. URL : http://journals.openedition.org/hel/914 ;

DOI : https://doi.org/10.4000/hel.914

\section{cc)}

HEL is licensed under a Creative Commons Attribution-NonCommercial-NoDerivatives 4.0 International License 


\title{
LINGUISTIQUE PSYCHOLOGIQUE ET SÉMIOTIQUE : LE CONTEXTE ALLEMAND ET SON INFLUENCE INTRODUCTION AU DOSSIER
}

\author{
Jean-Michel Fortis
}

CNRS, Histoire des théories linguistiques, Paris, France

Les articles qui composent le présent dossier sont issus du colloque SHESL qui s'est tenu à Paris du 25 au 27 janvier 2018, et dont l'intitulé était « La circulation $\mathrm{du}$ savoir linguistique et philologique entre l'Allemagne et l'étranger $\left(\mathrm{XVI}^{\mathrm{e}}\right.$ $\mathrm{XX}^{\mathrm{e}}$ siècles) ». Parmi les exposés, certains avaient une certaine proximité thématique qui nous a incités à les rassembler. On trouvera donc réunies ici des études consacrées à l'imprégnation psychologique et biologico-sémiotique de théories élaborées principalement au XIX ${ }^{\mathrm{e}}$ siècle et au sein du monde germanique, qui ont essaimé sous d'autres cieux (comme la Russie dans l'article de Sergueï Tchougounnikov, ou chez Van Ginneken dans celui de David Romand), non sans être réélaborées ni avoir connu des développements tangentiels en dehors de cette aire (Didier Samain rapproche à cet égard Uexküll et le behaviorisme). La discussion de Clemens Knobloch ouvre un horizon au-delà de la perspective psychologiste et représentationaliste, et évoque son dépassement en philosophie et linguistique.

Dans cette introduction, nous nous efforcerons de contextualiser les articles de ce dossier, autant que possible sans empiéter sur leurs contenus respectifs. Les trois premiers concernant la linguistique psychologique et son dépassement, nous décrirons d'abord le contexte qui permet de comprendre la montée de la perspective psychologique au XIX ${ }^{\mathrm{e}}$ siècle, et les spécificités de cet environnement allemand. Nous dirons ensuite quelques mots de la biosémiotique de Jakob von Uexküll, dans la seule fin de conduire le lecteur en amont et en aval de l'étude que lui consacre Didier Samain dans ces pages.

\section{LA MONTÉE DE LA PERSPECTIVE PSYCHOLOGIQUE}

La psychologie comme étude de l'âme, et la conception de l'âme comme principe vital de génération, croissance, sensation, intellection, ont un enracinement bien plus lointain que la période qui nous concerne ici. La nouveauté, à l'âge moderne, si l'on suit Vidal (2011), réside dans le fait que cette étude de l'âme, majoritairement conçue dans un esprit aristotélicien, en vient à se circonscrire à l'âme 
rationnelle, à la puissance intellective (mens ou animus) considérée séparément des facultés végétative, appétitive, sensitive et locomotrice. Une fois le domaine aménagé par cette spiritualisation acquis, il est tentant de jalonner l'évolution ultérieure par la constitution d'une sphère des idées chez Descartes et Locke, respectivement selon le critère de l'indubitabilité et l'intuition des "natures simples », qui tient encore à l'âme rationnelle, et d'autre part, sur le fondement de la présence immédiate des sensations à la conscience, c'est-à-dire par l'intégration des sensations au domaine de l'esprit. Cette évolution moderne se signale par le caractère fondationnel, pour une théorie de la connaissance, que revêtent les états mentaux chez Descartes et Locke. Comme le met en lumière Rorty, leur invocation d'un fondement dans la subjectivité les rapproche, en dépit de la différence des contenus (natures simples contre sensations) que les deux philosophes privilégient (voir Rorty 1990 [1979] : 70-75).

Tendent donc à se dissocier les considérations sur l'âme forme du corps et principe de vie organique, donc objet de la philosophie naturelle, comme chez Aristote, et celles sur l'esprit immatériel comme sphère des idées, situation dont découle le problème de l'interaction de l'âme et du corps (Vidal 2011: 78 et suiv.). Toutefois, l'agnosticisme, ou le scepticisme, de Locke quant à la notion de substance réelle, y compris celle de substance spirituelle, tend à neutraliser ce problème et favorise une attitude empiriste ou descriptiviste à l'égard des états mentaux: nous ne savons pas ce qu'est l'esprit mais nous décrirons ses opérations. L'empiricité définie par ce recadrage s'effectue aussi en délestant la psychologie de ce qu'on a parfois étiqueté comme « pneumatologie », c'est-à-dire les spéculations relatives à l'immortalité de l'âme, sa séparabilité du corps, sa création ou transmission, tous thèmes qui figuraient dans le premier ouvrage à s'intituler Psychologia, une anthologie compilée par Goclenius (1590).

La dualité de la pneumatologie et de la psychologie se reflète encore dans la dichotomie que Wolff établit entre psychologie rationnelle et empirique ${ }^{1}$. Mais tant dans l'œuvre de Wolff que dans sa réception, la partie empirique domine. Et de fait le XVIII ${ }^{\mathrm{e}}$ siècle témoigne d'une explosion des travaux affiliables à la psychologie empirique, certes sous des thématiques très diverses. Ainsi Vidal (2011 : 112-113) observe-t-il que les périodiques consacrés à l'anthropologie et à la psychologie connaissent, vers les années 1780-1790, une croissance exponentielle, tandis que l'entrée "psychologie » apparaît pour la première fois dans un catalogue bibliographique en 1778 (ibid. : 160).

1 On sait que Christian Wolff (1679-1754) était disciple de Leibniz. Feuerhahn (2002) soutient que la circonscription de la psychologie empirique résulte d'un abandon par Wolff du panpsychisme leibnizien, abandon qui met en regard cosmologie et psychologie : «[...] de manière surprenante, opposer deux types de réalités ontologiques ne conduit pas, chez Wolff, à opposer la psychologie à la cosmologie, une science finale à une science causale. Le dualisme ontologique, en tant qu'il attribue une autonomie aux éléments corporels, conduit à penser la science qui prend pour objet les monades spirituelles sur le modèle de celle qui connaît les éléments corporels. La "révolution" métaphysique quasi invisible de Wolff rend donc possible la naissance de la psychologie empirique qui s'ajoute ainsi à la traditionnelle psychologie rationnelle » (ibid. : 56). 


\section{PSYCHOLOGIE ET NATURALISATION DE L'ESPRIT}

Dans sa discussion de la distinction entre le normatif et le naturel, Hatfield (1990 : 14-16) fait intervenir plusieurs critères livrant, pour la période qui nous occupe ici, plusieurs définitions de l'attitude «naturaliste », ou naturalisante.

Une première forme (le naturalisme méthodologique de Hatfield) consiste à formuler des lois du psychisme dans des termes analogues à ceux des sciences physico-chimiques, par exemple, en faisant appel à une forme d'attraction entre idées, comme chez Hume (1978 [1739-1740]), ou de chemistry mentale chez Stuart Mill (1843 : 502). Cette analogie s'appuie éventuellement sur des protocoles soumettant les processus psychiques à des méthodes quantitatives, voire sur une quantification a priori des forces que constituent les représentations, comme chez Herbart.

Une deuxième forme de naturalisme sépare la réalité psychologique des idées de leur validité épistémique, s'abstenant ainsi de faire de la psychologie la pierre de touche de la connaissance vraie. Nous parlerons alors de naturalisme nonépistémique.

La dernière attitude naturaliste que nous distinguerons ici sous le nom de naturalisme réductionniste vise à réduire les opérations mentales, au moins partiellement, à des processus physico-chimiques, biologiques, ou neurophysiologiques.

Relèvent du naturalisme méthodologique les théories associationnistes qui, jusqu'au behaviorisme (chez Hull), invoquent régulièrement Newton (sans nécessairement être réductionnistes ni non-épistémiques). Pour Hatfield, Hume défend à la fois ce naturalisme et le délie de considérations sur la connaissance vraie ; Locke, en revanche, unit les deux, mais n'en est pas moins invoqué comme un des principaux « naturalisateurs » de la psychologie ${ }^{2}$. La psychométrie émergente est au même titre pensée sur le modèle des disciplines quantitatives, bien qu'au $\mathrm{XVIII}^{\mathrm{e}}$ siècle elle demeure largement prospective, spéculant sur les domaines d'application possible de la mesure (vouloir, sensations, affects, etc.; Vidal 2011 : 129-134).

Le naturalisme non-épistémique se présente chez un philosophe comme Herbart sous la forme d'une disjonction du logique, normatif, concernant les produits de la pensée, et du psychologique, qui embrasse les processus effectifs jouant entre les représentations, c'est-à-dire qui les lient, fusionnent, inhibent, etc. (voir Maigné in Herbart 2005 : 49-62). En reprenant des aspects de la pensée herbartienne, Steinthal (1855) entend lui aussi séparer logique et psychologique, espérant de la sorte ouvrir la voie à une naturalisation du langage, prise en charge par la psychologie.

Quant au naturalisme matérialiste, il peut être, au XIX ${ }^{\mathrm{e}}$ siècle, indépendant des précédents. Fechner, par exemple, prêche la neutralité ontologique de sa psychophysique : la psychophysique ne se prononce pas sur la question de la nature matérielle ou immatérielle de l'âme, même si Fechner lui-même embrasse une forme de panpsychisme (Nicolas 2002). Stuart Mill, qui propose un modèle associationniste de la liaison des idées, demande expressément que l'on distingue les états 
corporels et l'esprit qui sent (sentient mind) mais semble laisser ouverte la possibilité d'une réduction future de la psychologie à la physiologie (1843:497). Nous ne sommes cependant plus, ou de moins en moins, dans le cadre du dualisme de la res cogitans et de la res extensa : il s'agit plutôt de faire une distinction épistémologique entre deux ordres de phénomènes, en sauvegardant un type de discours (psychologique) portant sur la qualité et la quantité des sensations, et sur le contenu des représentations, tout en maintenant l'existence d'une corrélation nécessaire avec l'ordre "physique» au sens large (Romand 2010). Dans ses Vorlesungen, Wundt défend un monisme ontologique, mais un dualisme épistémologique entre le mental et le physique, c'est-à-dire l'impossibilité de définir le premier par le second (Wundt 1863), et de réduire la causalité à la causalité physique (Araujo 2016) ${ }^{3}$. Si le parallélisme est une thèse justifiée philosophiquement par Wundt, un linguiste comme Paul n'est pas si clair. Comme d'autres linguistes de l'époque, il sépare le physiologique, pertinent pour les lois phonétiques, du psychique, dont relèvent les rapports entre représentations (par exemple l'analogie), et qui ne relèvent que de l'organisme psychique, cette fois sans contrepartie « physico-physiologique » (voir par ex. Paul 1920 [1880] : 27-28). La coupure ontologique coïncide avec celle entre deux types de légalité linguistique.

Si l'on suit Freuler (1997: 173 et suiv.), ces formes de dualisme visent en partie à prolonger la survie de la philosophie en la rendant détentrice des investigations sur le sens interne. Les déclarations d'intention entendent satisfaire aux exigences de plus en plus impérieuses des sciences positives, sans toutefois permettre l'absorption de la psychologie dans la physiologie ${ }^{4}$. En dépit de cette résistance prolongée au réductionnisme matérialiste, il faut néanmoins constater que cette perspective s'affermit, non sans passer par quelques moments polémiques teintés de colorations politiques ${ }^{5}$. Les causes les plus facilement identifiables de cet affermissement sont à imputer à la crise de l'idéalisme en Allemagne, à l'expansion des sciences de la nature, en particulier de la physiologie, à la diffusion des sciences et d'un matérialisme populaire dans des textes bien relayés, y compris dans la presse (Freuler 1997 ; Morel 2017).

3 Ici s'insère l'hypothèse d'une énergie psychique spécifique de la pensée par analogie à l'énergie physique, dans le cadre d'un mouvement énergétiste qui s'oppose, dit Grot (1895), à l'atomisme du matérialisme scientifique. L'énergétisme a eu un certain succès en Russie, semble-t-il, et en linguistique il a été mis à profit notamment par Ovsianiko-Koulikovski (évoqué par S. Tchougounnikov dans ces pages). OvsianikoKoulikovski spécule par exemple que les moyens formels grammaticaux, en devenant des routines, passent dans l'inconscient, d'où résulterait une économie d'énergie, dont le surplus est libéré pour l'attention consciente et les « concepts » (Simonato 2005).

4 Entre autres voix, Auguste Comte abolit la psychologie dans son système des sciences. En Allemagne, Spiess (1844) la verse tout entière dans la physiologie (Freuler 1997 : 171-172). Contre les philosophes éclectiques qui accordent une place centrale à la psychologie, Broussais demande que la recherche sur « la causalité des phénomènes instinctifs et psychiques » soit l'apanage de la médecine (sur cette querelle française, voir Braunstein 2012).

5 Nous songeons en particulier à la « querelle du matérialisme » qui culmine en Allemagne en 1854, lors d'un congrès de la Société des naturalistes et médecins allemands, mais semble s'éveiller à partir des années 1840 (Morel 2017). Dans le domaine des sciences du langage, Max Müller, qui défendait l'identité de la pensée et du langage humains et était anti-darwinien, justifiait l'impossibilité du réductionnisme en agitant comme un repoussoir la célèbre phrase de Cabanis : non, le cerveau ne sécrète pas la pensée comme le foie sécrète la bile $(1873: 538)$. 


\section{APRÈS KANT}

Les formes de naturalisation du psychisme promues par la psychologie empirique se heurtent en Allemagne à l'idéalisme, et d'abord à Kant, qui rejette la scientificité de la psychologie pour la réduire à une enquête sur des faits anthropologiques $^{6}$. Sont fondamentales pour l'étude de la cognition humaine l'esthétique et l'analytique transcendantales, qui sont affaire de déduction, non d'observation empirique. La voix de Kant résonne jusque dans la théorie grammaticale, chez des auteurs comme August Bernhardi, Gottfried Hermann et August Grotefend. Ces deux derniers se servent par exemple des catégories kantiennes pour analyser la signification des cas (Burkard 2003), et on peut supposer que la floraison du localisme en Allemagne (Fortis 2018c) est au moins partiellement redevable à Kant de son questionnement sur le statut de l'espace, les interprétations psychophysiologiques qui font référence à la perspective transcendantale (chez Helmholtz), et les contre-réactions empiristes (comme chez Herder dans sa Metakritik). Ces contre-réactions ont été bien documentées dans l'ouvrage de Formigari (1994), qui montre en outre comment des conceptions empiristes réévaluent, à l'encontre de Kant, la place du signe dans l'acte de connaissance, établissant généralement une continuité entre la sensibilité, l'imagination, la mémoire et l'activité linguistique, sans toutefois identifier pensée et langage. Cette dépendance relative de l'une à l'égard de l'autre se reflète aussi dans des réflexions qui relèvent de la Sprachkritik?.

C'est un leitmotiv commun aux ouvrages de Beiser (2014) et Freuler (1997) que des réinterprétations de Kant à tendance empiriste semblent ne s'imposer vraiment qu'à la suite du reflux de l'idéalisme, toujours dans l'arrière-plan d'une montée en puissance des sciences de la nature. Chez Herbart, la psychologie élève des prétentions à devenir scientifique, en prenant le contrepied de thèses idéalistes : le Moi n'est plus l'origine de la déduction et de l'empirie comme chez Fichte, mais il est pour ainsi dire le centre géométrique de représentations associées; les facultés de l'âme sont abolies ; une statique et une mécanique visent à expliquer et même quantifier (quoique par des raisonnements $a$ priori) les degrés de liaison et d'inhibition entre représentations envisagées comme forces ; enfin, la tendance de l'activité représentative à conserver sa simplicité justifie le fait que la conscience constitue une sorte de goulot d'étranglement pour des représentations qui ne peuvent être «aperceptées » qu'en dépassant un certain seuil. Ce dépassement est favorisé par l'intégration de la représentation active à une «masse » existante de représentations. La thématique des groupes de représentations inconscients unis par des liens associatifs est ainsi introduite.

Elle aura un succès certain en linguistique, surtout sous la forme que lui donnera Steinthal (1871). Ainsi, les groupes associatifs de Hermann Paul (1920

6 Un locus classicus est la préface des Premiers principes métaphysiques de la science de la nature (Kant 1985 [1786] : 368-369).

7 C'est-à-dire d'une critique du langage comme source d'erreurs et d'illusions. Cette critique atteste d'une foi dans le pouvoir du langage, mais aussi de la possibilité de s'en déprendre. Parmi les auteurs considérés par Formigari, le thème est très présent chez Reinhold mais se rencontre aussi chez Herbart. 
[1880]), qui emploie la terminologie de Herbart et Steinthal, seront vraisemblablement repris par Saussure (Samain 2014). Le cadre herbartien est aussi étendu à la synthèse prédicative : celle-ci réside dans la fusion avec une masse de représentations et cette fusion est ce qui remplit la fonction de la copule (Steinthal 1855 : 324-325). En tant que corrélat du mot, la représentation (Vorstellung) est comme une grandeur nulle qui accède à une position du jugement. Si elle apparaît comme sujet, elle est en attente de détermination ; mais en même temps elle donne accès aux traits (Merkmale) venant de l'intuition ; c'est une mendiante qui cache un trésor de prédicats (ibid. : 334-335). Cette perspective psychologisante paraît associée, chez Steinthal, aux prémices d'une réflexion sur la discordance entre le sujet et le prédicat grammaticaux et d'autre part, pour emprunter le vocabulaire de l'époque, le sujet et le prédicat psychologiques. Selon Elffers (1999), Steinthal aurait embrayé cette discussion qui, au $\mathrm{XIX}^{\mathrm{e}}$ siècle, se révéla particulièrement fertile ${ }^{8}$.

L'article de Sergueï Tchougounnikov développe les ramifications qu'a eues en Russie cette perspective psychologisante sur la relation prédicative.

Notons que la psychologie de Herbart est articulée à une métaphysique qui n'intéresse pas Steinthal, et que Wundt considérera comme un fondement douteux de la thèse de la simplicité de l'âme. Quant à la conception que se fait Herbart du langage comme instrument de communication et de manipulation de l'action d'autrui, elle est exécrée : Steinthal, et Misteli à sa suite, parlent avec mépris de ce qu'ils assimilent à la thèse de l'invention (et pourtant Herbart n'est pas un tenant de l'invention). Que la langue ne soit pas «das bildende Organ des Gedanken [sic] », selon l'expression de Humboldt (1836: 56), apparaissait aux yeux de Steinthal dépassé et inenvisageable (Knobloch $1988: 163-164)^{9}$. Le butin que Steinthal rapporte de sa fréquentation de Herbart consiste donc essentiellement en sa théorie psychologique et sa vision de la logique.

\section{LA LINGUISTIQUE PSYCHOLOGIQUE ET SES ENJEUX}

Steinthal joint donc un élément idéaliste humboldtien et romantique (celui de l'âme de l'homme se conformant ses moyens d'expression) avec un appel à une forme de naturalisme méthodologique et non-épistémique, par le biais de la psychologie. Ce naturalisme est en outre étayé par sa théorie de l'origine, fondée sur l'association et le réflexe, notion qu'il dit avoir eu l'idée d'intégrer à sa théorie en lisant le Handbuch der Physiologie de Johannes Müller (1834-1840 ; Steinthal $1855: 257)^{10}$.

Cet ensemble de thèses, dont la combinaison peut étonner le regard contemporain, dessine ou fait pressentir les enjeux de la linguistique psychologique d'origine allemande dans la période que nous considérons ici. C'est en nous

8 Mais voir aussi notre note 10 . L'analyse à notre connaissance la plus détaillée de ces débats se trouve chez Elffers (1991). L'article de 1999 en présente un aperçu.

9 «L'organe qui donne forme au contenu de la pensée », dans la traduction de Humboldt par Caussat (Humboldt 1974 [1836] : 192). «Organe formateur de la pensée » serait plus neutre.

10 Ce qui ne l'empêche pas de considérer le matérialisme comme une abomination (1855:274-275). 
appuyant sur la somme que leur a consacrée un des auteurs de ce dossier, Clemens Knobloch (1988), que nous tenterons de circonscrire les enjeux en question. Bien sûr, nous ne pouvons faire pleinement justice à cet ouvrage dans ces quelques lignes, et des simplifications sont inévitables. Voyons les principaux axes autour desquels oscillent les positions des acteurs.

Nous y avons fait allusion, l'association établie entre la thèse de l'invention plus ou moins concertée du langage et une conception pratique de celui-ci comme instrument de communication est prise à contrepied chez Steinthal : le langage est plutôt un moyen d'exprimer l'intériorité émanant d'une pulsion à convertir en signes un contenu psychique, qui est d'abord un sentiment (Gefühl), puis un trait (ou caractère, Merkmal, le terme de Herder, 1978 [1772]) pris sur l'intuition de l'objet, avant de se conventionaliser. Les strates qui s'étagent de l'intuition d'un caractère perceptif au signe sans corrélat intuitif sont situées le long d'un parcours historique fictionnel, c'est-à-dire décrites dans une théorie de l'origine du langage. Steinthal est loin d'être isolé et les théories de l'origine prolifèrent : un auteur comme Noiré (1877) formule une théorie socio-psychologique de l'origine qui met en exergue la coordination requise par le travail en commun et rejette l'idée de signes sonores qui auraient crû par la force de la nature, à titre de réactions spontanées. Cette dernière thèse sert à Marty (2017 [1875]) de principe de classification dans son récapitulatif des théories de l'origine: ceux qui l'adoptent sont classés comme nativistes, ceux qui la rejettent comme empiristes.

L'opposition entre la conception expressive, représentationaliste et individualiste, d'une part, et la conception communicationnelle n'est pas absolue et l'on voit certains auteurs adopter des positions intermédiaires. Marty, prolongeant un point de vue dix-huitièmiste, réintroduit la communication intentionnelle (absichtliche Mitteilung) comme moteur principal de la création du langage, de son développement et de son progrès. Mais il demeure en quelque sorte à michemin de Wundt et Bühler : il analyse le langage comme manifestation (Kundgabe) de la vie psychique, mais aussi comme un ensemble de fonctions communicatives (Knobloch 1988: 154-155, 274-280). Paul illustre une autre forme d'ambivalence entre ces points de vue communicationnel et représentationaliste : il définit le « sujet psychologique » comme la masse aperceptante (suivant en cela Steinthal) mais aussi comme le thème du discours, approuvant ainsi Gabelentz de caractériser le sujet, du point de vue de l'auditeur, comme ce sur quoi l'attention de ce dernier est attirée (Knobloch $1988: 218-219)^{11}$.

La distinction entre les relations conceptuelles logiques et la conformation linguistique de la pensée est thématisée chez Steinthal par la notion de forme interne spécifique à une langue. Le point de vue dominant sur la structure sémantique et grammaticale est relativiste, même dans le cadre d'une théorie psychologisante. Maintenant, des vestiges de l'analyse logique demeurent (Knobloch

11 Knobloch (1988) voit en Weil (1844) la source d'un point de vue dual sur la syntaxe, en tant qu'elle refléterait à la fois des modes de conceptualisation des événements et ce que nous appelons aujourd'hui une structure informationnelle. On peut penser que la prise en compte de cette structure suit des discussions sur le facteur « émotionnel » dans l'ordre des mots et l'ordre «naturel» ou réflexif. Ricken (1969) pense que le sensualisme de Condillac a désamorcé le conflit entre ces ordres. Il aurait ainsi contribué à faire pénétrer la perspective communicationnelle dans la syntaxe. 
1988 : 402-404). De Heyse à Wundt, la décomposition de la phrase en jugements simples rappelle fortement l'analyse des énoncés à sujet et attribut complexes dans la grammaire de Port-Royal, analyse qui reflète une pénétration de la logique dans la grammaire et dont une des visées était d'adapter les énoncés à la syllogistique (Raby 2018, chap. 2 et 3). Sur ce point, Wundt est exemplaire : il propose une décomposition psycho-logique de la phrase en jugements simples (paires sujet-prédicat) sous une forme arborescente. Après Knobloch (1988 : 433435), Graffi (2001 : 136-137) note que la description de Wundt rappelle l'analyse de la proposition en divisions successives proposée par Becker, analyse décomposant elle aussi les membres de la proposition en relations conceptuelles (Becker 1841 [1827] : $230 ; 1843: 6$ et suiv.). La proximité entre Wundt et la grammaire générale semble peu douteuse ${ }^{12}$. Paradoxalement, on peut penser que ce genre d'arborescence a influencé la future analyse en constituants, par l'intermédiaire de Bloomfield (Percival 1976 ; Seuren 1998). Mais cette analyse supposait que l'on rompît avec cette psycho-logique et qu'on revînt aux formes de surface.

La naturalisation individualiste promue par la psychologie entre en tension avec les notions employées pour constituer et décrire des entités supra-individuelles, comme l'esprit du peuple, que Steinthal semble hypostasier sous la forme d'une nouvelle individualité. Paul, en revanche, fera de la langue commune (Gemeinsprache) une abstraction faisant office de norme idéale; seul l'esprit de l'individu est réel (Paul 1920 [1880] : 404-405). La distinction entre l'individuel et le supra-individuel donne lieu chez Wundt à la bifurcation entre la psychologie expérimentale, qui traite de l'individuel, et la Völkerpsychologie, ou «psychologie ethnologique » dans la traduction de Ribot (2003 [1879]). Cette dernière discipline traite, négativement, de toutes les productions spirituelles qui ne peuvent être l'œuvre d'une «conscience individuelle» (Wundt 1920: 218), et positivement, des stades de civilisation au travers des langues, mythes et coutumes des peuples (Wundt 1912a). Le langage et la diversité linguistique sont donc traités dans la Völkerpsychologie. Toutefois, comme Wundt rétablit l'identité de la grammaire, de la logique et de la psychologie, des analyses de la Völkerpsychologie trouvent leur double cognitif dans la partie logique de l'œuvre. Il en va ainsi de la théorie des cas, qui a son correspondant cognitif sous la forme d'une typologie des liaisons conceptuelles. De même encore, l'analyse de l'énoncé en propositions simples (Wundt 1912b : 331) a sa contrepartie exacte dans la Logik en tant que principe de division binaire des représentations, qui est censé fonder la dualité du sujet et du prédicat (Wundt $1880: 53-54$ ). S'il fallait trouver une théorie des cas ayant une dimension supra-individuelle, par exemple fondée sur des contraintes d'ordre communicationnel, on devrait se tourner vers Marty plutôt que vers Wundt. Conformément à la perspective générale de Marty sur le rapport entre la forme interne (soit la conformation linguistique de la pensée) et la signification visée, la première est un instrument permettant de communiquer la seconde, non un reflet de cette dernière ou de la cognition, 
comme elle le serait chez Wundt, que Marty ne perd pas une occasion de vilipender ${ }^{13}$.

Ce dernier point nous conduit au dépassement de l'idée que la compréhension de la signification des formes linguistiques est l'activation psychique de représentations (ou de rapports entre représentations). Cette question est un des objets de l'article de C. Knobloch dans ce numéro, mais elle a été aussi abordée dans son ouvrage de 1988, où il identifie un groupe d'auteurs ayant joué un rôle important dans ce revirement. D'une manière ou d'une autre, explique-t-il (1988 : 379-380), Madvig, Bréal, Svedelius, Marty et Wegener ont en commun d'envisager les signes linguistiques comme des moyens en vue de l'intercompréhension, non comme des étiquettes de représentations. Se dessine ici une perspective fonctionaliste, qui explique des faits structurels par la satisfaction de fonctions utiles à la communication. Pour Madvig (1875 [1856] : 93 et suiv., 104 et suiv.), par exemple, doit être grammaticalement marqué précisément ce qui ne se laisse pas supposer ou déduire des conditions habituelles de la communication. La structure communicative sujet-prédicat n'a pas à être marquée, car il est toujours présupposé que le locuteur veut dire quelque chose de quelque chose. Mais pour inhiber l'interprétation prédicative et close de Mann lauf (c'est-à-dire der Mann läuft, «l'homme court ») et en faire une structure susceptible d'intégration, il faut un moyen grammatical : d'où ein laufender Mann (littéralement, « un homme courant »).

Lorsque le résultat de l'interprétation est régulièrement intégré au signe, il constitue son idée latente, dit Bréal (1868), qui fournit des illustrations assez similaires à celle de Madvig citée à l'instant. En devenant élusive, l'idée latente ne permet plus d'attribuer au signe une signification univoque, ce qui entraine une suppléance éternelle des signes par le «travail intellectuel» (selon l'expression de Bréal), c'est-à-dire le calcul de l'interprétation. Dans cette perspective, on ne peut attendre que les outils grammaticaux et les formes lexicales puissent faire plus que jalonner l'interprétation; ils n'enferment pas en eux virtuellement le contenu intenté.

\section{REPRÉSENTATION ET SENTIMENT ( GEFÜHL)}

À une époque où l'activité consciente est un objet essentiel de la psychologie, si ce n'est l'objet unique de la psychologie individuelle, comme chez le dernier Wundt, la notion de sentiment permet de fonder, compléter ou même remplacer la représentation en tant qu'état psychologiquement réel associé aux formes linguistiques.

13 En voici un exemple. Les cas témoignent-ils d'un privilège cognitif des déterminations sensibles (spatiales, dans l'hypothèse localiste) ? Non, dit Marty, car c'est une loi générale que « les représentations concernées [i. e. celles relatives à la forme interne] - en particulier lorsqu'elles ont simplement pour but l'intercompréhension - sont empruntées de façon privilégiée au domaine de la sensation, de la réalité physique, et en particulier de la réalité visible » (Marty 1910 : 103). Ces percepts, parce qu'ils sont partagés dans l'expérience commune, facilitent la communication de significations (plus « abstraites ») qui peuvent être difficiles à transmettre. Le caractère systématique, chez Wundt, d'un parallélisme entre formes et contenus conceptuels est nuancé par C. Knobloch (à propos de la théorie des cas ; 1988 : 374-375). 
C'est un acquis de la recherche de David Romand d'avoir montré l'importance de la thématique du sentiment et de ses ramifications dans la période embrassée ici, particulièrement entre 1850 et 1910 . Dans son article du présent numéro et dans des travaux antérieurs (Romand 2015, 2018), D. Romand met en exergue le rôle de Herbart et de ses successeurs, notamment sur deux points : (1) la thèse du caractère dérivé du sentiment à l'égard des représentations, dont il manifeste un rapport, ou exprime la qualité de la transition qui les lie ; (2) la constitution d'une typologie qui va bien au-delà de la dichotomie du plaisir et du déplaisir (Lust/ Unlust) et outrepasse Herbart.

La catégorie des sentiments formels de Waitz, dont l'archétype est chez lui le sentiment d'attente, a chez Van Ginneken son pendant dans le sentiment de connexion, comme le note D. Romand ici. C'est la relation associative entre des représentations qui est le support de ce sentiment. Une fois « réfléchi» sur un mode conceptuel, ce sentiment peut donner lieu à des relations opposées : wieder 'de nouveau' établit une égalité, wider 'contre', une inégalité, mais tous deux sont fondés sur une connexion par ressemblance (Van Ginneken 1907 : 135-138). On trouvera chez William James, mentionné d'ailleurs par Van Ginneken, un développement parallèle sur les feelings of relation, qu'il associe aux mêmes parties du discours que Van Ginneken (les prépositions, conjonctions et adverbes; James 1890: 245). Chez James, il s'agit de trouver un corrélat conscient à l'emploi de mots qu'une certaine tradition, qui les englobait sous le nom de «particules », considérait comme renvoyant à des actes ou manières, et non à des concepts (voir Nuchelmans 1986). Il s'agit en fin de compte d'assigner un corrélat sémantique conscient à des mots auxquels on ne trouve pas de contenu représentationnel, soit parce que leur origine sémantique est très indéterminée (chez Van Ginneken), soit parce que ces mots ayant été à tort regardés comme signifiant des actes, la saisie subjective de leur apport sémantique devient difficilement descriptible pour une psychologie du vécu (chez James).

Comme phénomène survenant à des rapports entre représentations et portant cette fois sur leur contenu propre, Waitz avait isolé la catégorie des sentiments qualitatifs (qualitative Gefühle) accompagnant par exemple la saisie d'une démonstration ou l'appréhension de la rectitude de formes linguistiques (Gefühl der (Un)richtigkeit; Waitz 1849 : 309-311, 336-340 ; voir Romand 2015). Progressivement se légitime, à une époque où l'activité consciente est un objet essentiel de la psychologie, la notion de sentiment « intellectuel » entendu comme émanation consciente d'un rapport et d'un système de rapports entre représentations. On voit l'application qui peut être faite à la sémantique, et dont Van Ginneken nous offre de nombreuses illustrations. Avec Gomperz, il semble être l'auteur qui a le plus exploité la notion de sentiment en matière de recherche sur le langage.

Il est intéressant d'évoquer ici la contrepartie esthétique des sentiments intellectuels. L'œuvre d'art peut en effet être conçue elle aussi comme un système de rapports, dont l'architecture offre l'exemple peut-être le plus palpable. Déjà chez Winckelmann, nous voyons émerger une conception « formaliste » du plaisir esthétique, séparé du plaisir sensuel et des passions, et dévalorisant les soi- 
disantes vertus de l'imitation pour insister sur les proportions et les formes (Barasch 1990 : 120-121 ; voir Winckelmann 1764, chap. 4, section 2). Waitz et Nahlowsky séparent de même la satisfaction sensuelle du plaisir esthétique, que le premier associe aux formes spatiales et temporelles, tandis que le second parle de l'œuvre d'art comme d'un système de relations (Romand 2015; Romand 2018). Ces variétés de formalisme vont rencontrer un courant psychologique de l'esthétique qui, à partir, semblerait-il, de Vischer (1873), explique l'émotion artistique par la projection d'affects empathiques sur les formes: tel rocher imposant évoque un sentiment de défi, tel horizon qui s'épanouit suscite un sentiment de triomphe, etc. Cette conjonction est perceptible chez Lipps (1897), chez qui le sentiment de la forme (Formgefühl) suscite à la fois une sorte d'élargissement du moi par projection empathique, et résulte de l'articulation du multiple en un tout ou de la saisie de règles inconscientes. Ce dernier aspect justifie de le rapprocher du sentiment de la langue (Sprachgefühl; Lipps 1907). Nous sommes ici en présence d'une autre manière de concevoir le rapport entre sentiment et langage : non comme un contenu sémantique de nature affective (à l'instar de Van Ginneken et Gomperz), mais comme pointe consciente d'un système sous-jacent. Cette conception sous-tend la manière qu'a Sapir de théoriser la saisie intuitive de l'organisation d'un système de formes, saisie qu'il nomme form-feeling (Fortis 2015, 2019).

\section{LE DÉPASSEMENT DE LA CONCEPTION REPRÉSENTATIONALISTE}

Les descriptions affectivistes offrent une alternative à la conception représentationaliste. Sous sa forme caricaturale, cette dernière reconduit la triade sémiotique vox-conceptus-res, dans laquelle le « concept » est image de la chose et signification du mot. Cette version simpliste est néanmoins complexifiée par des tenants du représentationalisme. Chez Steinthal, par exemple, la représentation (Vorstellung, associée au mot) est comme une grandeur nulle qui accède à une position du jugement. Si elle apparaît comme sujet, elle est en attente de détermination ; mais en même temps elle donne accès aux caractères venant de l'intuition. Comme elle permet à l'intuition d'entrer dans le jugement et que l'emploi d'un mot dans de multiples contextes judicatifs enrichit la signification de ce mot, elle permet à la signification du mot de se déployer et de s'enrichir (1855:334-335).

Van Ginneken admet fort bien que les mots ne sont associés « mécaniquement » à des représentations intuitives que dans les cas les plus simples : il en va ainsi, chez l'enfant, explique-t-il (1907 : 22). Mais bien souvent, aucune intuition n'est corrélée à l'emploi des mots, et à la limite ne reste plus à l'état conscient qu'une sensation (ou représentation potentielle), qui est toutefois susceptible de donner accès à des déterminations du référent (1907 : 27-28). La discussion de Van Ginneken sur les représentations non intuitives et ses analyses affectivistes témoignent de multiples accointances avec des thèmes classiques ou propres à l'époque : le thème de la langue comme calcul aveugle, celui du discours intérieur, les réflexions de philosophes de l'école de Brentano sur la non-immanence de l'objet représenté, celui, très français, de la pathologie et des idiosyncrasies 
individuelles qui rencontre les études « expérimentales » sur les représentations ou leur absence lors de tâches linguistiques. Cette dernière approche semble initiée en France par Binet, dont la méthode introspective sera reprise par les psychologues de l'école de Würzbourg. Lorsque Binet demande à sa fille Armande ce que le mot tempête évoque en elle, celle-ci répond: «je ne me représente rien » (Binet $1903: 84$ ).

Comme nous venons d'y faire allusion, en France, le thème de la " pensée sans images » croise celui du discours intérieur et des particularités individuelles (sujets imageants, sujets visuels, auditifs, etc.), des parafantaisies (Dugas 1895), c'est-à-dire des images parasites, non « adaptées à l'idéation » dit Binet, de la pathologie enfin ${ }^{14}$. Dans l'aire germanophone, la pensée sans images est déclinée en états vécus associés à des tâches de résolution de problème. Cette orientation est par excellence celle de l'école de Würzbourg. Ach (1905), par exemple, identifie sous le terme de Bewusstheit une conscience inchoative de l'opération à effectuer ou de son résultat, mais aussi de la signification d'un mot. La perspective est encore partiellement représentationaliste, dans la mesure où la Bewusstheit de la signification est attribuée à des structures nerveuses sous-tendant la formation d'images associées au mot, mais sous-activées. Cette conception revient à dissocier la représentation consciente de la signification des représentations qui constituent la signification. La représentation consciente de la signification est décrite comme l'état vécu suscité par la "potentialisation » (Bereitschaftsetzung) des représentations qui constituent la signification, décrite de façon associationniste (ibid. : 217) ${ }^{15}$.

Le dépassement du représentationalisme porté par ces infléchissements est corrélatif de la querelle du psychologisme qui sévit en Allemagne au tournant des $\mathrm{XIX}^{\mathrm{e}}$ et $\mathrm{XX}^{\mathrm{e}}$ siècles et qui porte essentiellement sur la réductibilité des lois logiques à des régularités psychologiques. Quoique le débat ait impliqué de nombreux participants, c'est la figure de Husserl qui finit par dominer, du moins une fois que Scheler l'adoube après la guerre comme vainqueur du psychologisme, dans un climat propice à des idées anti-positivistes, holistes, existentialistes (Kusch 1995 : 256-257). La controverse reflète aussi une réaction à l'accaparement de chaires de philosophie par des psychologues qui sont parfois des expérimentalistes « durs », comme Ebbinghaus. Le comble de l'hostilité est atteint quand, en 1913, 107 philosophes d'Allemagne, Autriche et Suisse signent une pétition exigeant de ne plus attribuer de chaires de philosophie à des psychologues.

La querelle du psychologisme et Husserl nous mènent à la perspective noétique discutée par $\mathrm{C}$. Knobloch dans son article. Est noétique, d'après la définition soumise (section 3), une conception selon laquelle les énoncés en situation de parole ont un contenu intenté qui n'est pas lui-même un énoncé, qui n'est pas un contenu de conscience (ou une représentation au sens du sensualisme

14 «La psychologie française fin de siècle apparaît au plan international comme une psychologie pathologique » (Carroy et al. 2006 : 59). Le chapitre 3 de cet ouvrage développe un panorama du " modèle pathologique » dans la psychologie française. Représentatif à cet égard, Delacroix (1924) offre une synthèse des recherches sur les hallucinations, l'endophasie et l'hyperendophasie, les aphasies, etc.

15 L'ouvrage de Humphrey (1951) contient un bon exposé critique des recherches de l'école de Würzbourg. 
du XIX ${ }^{\mathrm{e}}$ siècle) et qui n'est pas non plus la chose désignée. Une telle conception est antipsychologiste dans la mesure où le contenu intenté reste identique à luimême en dépit de la variation des contenus psychiques associés, ce qui s'applique à la notion de signification telle que Husserl l'analyse dans ses Recherches Logiques (1959 [1900]). Mais comme C. Knobloch le montre, la conception noétique peut aussi caractériser la théorie de la signification de Gomperz (1908) dès lors que la signification y est séparée de la conceptualisation de la situation à laquelle elle fait référence. Enfin, en suivant le fil conducteur de la conception noétique déroulé par C. Knobloch, celle-ci nous conduit, au-delà de Husserl et Gomperz, à la perspective pragmatique de Gardiner (1932), selon laquelle le contenu intenté (thing-meant) est hors du langage mais présenté par lui, et objet de négociation dans la situation de parole. Ici se trouve confirmé le potentiel antireprésentationaliste de ce que nous avions identifié plus haut comme le point de vue communicationnel et fonctionaliste.

\section{UEXKÜLL ET LA SÉMIOTIQUE}

Le dernier article de ce dossier organisé chronologiquement concerne la figure la plus récente, Jakob von Uexküll. Comme pour la linguistique psychologique, nous tenterons de présenter le cadre intellectuel général dans lequel s'insère historiquement le biologiste.

\section{Position historique d'Uexküll}

La perspective théorique d'Uexküll peut être commodément résumée par la formule de « vitalisme sémiotique ». Son vitalisme réside dans l'irréductibilité du rapport entre organisme et Umwelt à des facteurs physico-chimiques, ainsi que dans la croyance à une force vitale dirigeant la croissance du vivant, sans préformation ni assemblage partie par partie ${ }^{16}$. Sur ce dernier point, Driesch, qui travaillait sur la parthénogenèse et la régénération, est peut-être son autorité majeure. Notons que la position d'Uexküll n'en fait pas un excentrique : Bergson, tant lu à l'époque, a sa place dans l'Histoire du vitalisme de Driesch au côté de l'auteur lui-même (1922), et il existe dans l'aire germanophone un solide courant vitaliste dont émane d'ailleurs une terminologie qui circule, à l'époque romantique, entre philosophie et linguistique. Ainsi la Bildungstrieb de Blumenbach, concept biologique anti-préformation, se retrouve-t-elle chez Humboldt à propos cette fois des langues.

Quant à l'aspect sémiotique de sa théorie, il a lui aussi des antécédents. Uexküll salue Helmholtz pour avoir le premier identifié les qualia de sensations à

\footnotetext{
16 Nous renvoyons à l'article de Didier Samain pour une présentation du concept d'Umwelt. Sebeok traduit Umwelt par self-world, subjective world/universe. Gipper (1963 : 377-381) esquisse une analyse du champ sémantique d'Umwelt (qui contraste avec Umgebung, Außenwelt, Eigenwelt/Erscheinungswelt, Innenwelt et bien sûr Merkwelt et Wirkwelt ; ces deux derniers concepts et celui d'Umgebung sont expliqués dans le même article de ce numéro). Comme Didier Samain, nous conservons Umwelt. Il est plus que délicat de traduire Umwelt par milieu, non seulement parce qu'Uexküll les distingue, mais aussi en raison des implications politiques et nationalistes du terme de milieu (Feuerhahn 2009). Harrington note qu'Uexküll et ses partisans eurent à se défendre de l'assimilation de l'Umwelt au milieu, concept déterministe qui, pour les Nazis, pouvait fleurer le bolchevisme (Harrington $1996: 69$ ).
} 
des signes des événements cérébraux, et par leur intermédiaire, des événements du monde extérieur (1902: 17). Comme chez Helmholtz, le point de vue d'Uexküll est kantien : le monde subjectif est infranchissable et la chose en soi inaccessible ; et comme chez Helmholtz, la notion de signe abolit le mystère, évoqué par Kant, d'une pensée consciente inétendue qui peut se projeter dans l'espace de l'intuition (ibid. : 16-17). Notons que Lotze parlait déjà de « signes locaux » (Lokalzeichen) à propos de sensations coordonnées à des nerfs moteurs dirigeant le regard vers des points de fixation donnés, le tout dans le cadre d'une métaphysique dualiste (Lotze 1852) ${ }^{17}$.

\section{Les médiateurs}

Il semble que la stature d'Uexküll comme source fondamentale de la biosémiotique (ou de la "biologie théorique») se soit établie par deux médiations principales : les sémioticiens, écologues et éthologues de Tartu, en Estonie, d'où Uexküll est originaire; et dans le monde anglophone, par l'intermédiaire de Sebeok. En Estonie, selon le témoignage de Kull (2001 : 13-14), Uexküll a été redécouvert dans les années 1970 au travers des écrits de Konrad Lorenz, donc par la voie de l'éthologie, pour devenir la référence majeure des biosémioticiens de Tartu. On peut supposer que la mémoire de la lignée Karl von Baer-Uexküll, tous deux Estoniens et d'inspiration vitaliste, n'y avait pas succombé à l'expansion globale du darwinisme et que l'environnement était favorable ${ }^{18}$.

Dans le monde anglophone et au-delà, et plus tard en interaction avec les chercheurs de Tartu, la diffusion de la perspective zoosémiotique d'Uexküll a été assurée, nous l'avons dit, par Sebeok ${ }^{19}$. Sur la foi d'une brève rétrospective rédigée par l'auteur lui-même (Sebeok 1979: 193), la première mention d'Uexküll apparaît chez Sebeok dans un compte rendu de 1963 publié dans Language, dans le contexte d'une discussion de systèmes de communication animale. Soit par pédagogie (l'auteur s'adresse à des linguistes), soit qu'il soit venu lui-même à la question par ce biais, Sebeok commence par le familier, c'est-à-dire les illustrissimes travaux de von Frisch sur les abeilles, et décrit succinctement leur réception proto-biosémiotique chez des linguistes, en l'occurrence Bühler, Kroeber, Laziczius, Lotz qui propose une synthèse «structurale » (sous forme d'oppositions sémiques) des résultats de von Frisch, Benveniste qui, selon Sebeok, reprend Lotz, et enfin Hockett ${ }^{20}$ (Sebeok 1963 : 448-449).

17 Le terme de Lokalzeichen est repris par Uexküll (1902: 7, et entre autres dans les Streifzüge), sans mentionner Lotze cependant. Comme Helmholtz (1867) l'a repris avant lui, et que Uexküll lisait Helmholtz, il est vraisemblable que ce dernier est la source d'Uexküll. Sur la théorie des signes locaux, voir Fisette (2015). Notons que l'Écossais Thomas Reid proposait déjà une théorie sémiotique de la perception de la distance, taille et forme des objets (1997 [1764], chap. VI, § 22-23). Kuehn (1987) explore l'influence de Reid en pays germanique, en particulier chez Tetens, qui reprend la théorie sémiotique de la perception de Reid.

18 Sur cette lignée dans le contexte estonien, voir Brentari (2015:24-25).

19 Et par l'International Association for Semiotic Studies et la revue Semiotica, créées en 1969.

20 Chez qui il s'agit, comme chez Martinet et Benveniste, de spécifier ce qui sépare le langage humain des systèmes de communication animale. Le critère de la double articulation apparaît quasi simultanément chez Martinet et Hockett (qui parle de duality). Voir notamment Hockett (1958 : 574 et suiv.). 
À la différence d'un Hockett, il n'est plus question chez Sebeok d'accorder un statut exceptionnel au langage humain. Il s'agit bien plutôt, en exploitant (entre autres) la mise à jour des résultats de von Frisch par son disciple Lindauer, et l'image plus complexe de la communication apiaire qui en résulte, d'insister sur la diversité et la sophistication de la communication animale et de la rapprocher de celle des humains. Rôle mnémonique des signes, évolution diachronique et diatopique (des systèmes apiaires par exemple), présence de signaux métacommunicatifs sont autant de traits qui, explique Sebeok, sont attestés chez les animaux et comblent partiellement le fossé (Sebeok 2001 [1994]). Enfin, depuis ces premières années de militantisme, la biosémiotique a prospéré en unissant les forces de biologistes, éthologues, linguistes et philosophes ${ }^{21}$. Une troisième voie, plus secondaire, de l'acclimatation d'Uexküll chez les linguistes est restée plutôt confinée à l'aire germanophone. On peut la décrire comme le rapprochement entre la notion biologique d'Umwelt et l'idée d'une construction linguistique du monde, d'une sorte d'Umwelt verbale, chez un néo-humboldtien comme Gipper. Ce rapprochement est mis à l'épreuve de la thématique philosophique de l'ouverture au monde chez l'homme (sa Weltoffenheit dit Max Scheler, 1928), sa déprise de l'Umwelt, qui questionne la possibilité, justement, d'étendre la notion d'Umwelt à l'espèce humaine. L'ouvrage de Gipper (1963) se fait l'écho de ces débats, notamment de la question de savoir si le monde de la culture et le langage peuvent être ramenés sous cette notion.

\section{L'acceptation de Uexküll}

Nous ne sommes plus, dans les années 1960 et 1970, à une époque où discuter de la communication animale nous enferre dans la question du degré de conscience des animaux, ou, par exemple, de la question de savoir s'il faut accorder une «activité psychique» ou une "vie intellectuelle» aux fourmis (voir Romanes 1883, suivi par Ribot 1919, pour une conception « intellectualiste », en termes de facultés de mémorisation et de généralisation). L'approche comportementaliste visant à effacer toute analogie à l'expérience vécue humaine et expurger la biologie de sa terminologie anthropomorphique, défendue par Uexküll précocement (Beer, Bethe \& Uexküll 1899), a permis de neutraliser ce questionnement, non sans rencontrer des résistances : Claparède (1902) classe Bethe et Uexküll dans la catégorie suspecte des "ultra-physiologiques » qui veulent la fin de la psychologie comparée. Nous touchons ici à la question du rapport d'Uexküll au behaviorisme. Dans son étude (ce numéro), Didier Samain montre bien que les aspects behavioristes de la pensée d'Uexküll ne caractérisent pas seulement cette première phase de ses travaux.

Entre-temps, le métalangage sémiotique a bénéficié de la mathématisation du concept d'information, et a été étendu au système immunitaire ou au code génétique. Un autre obstacle a été plus ignoré que levé : celui d'un discours enraciné dans la science romantique, aux accents goethéens, si frappants chez Uexküll,

21 L’ouvrage collectif dirigé par Favareau (2010) propose une histoire du champ, un panorama de ses divers domaines et de ses contributeurs principaux. 
quand il dit par exemple, observant la parfaite coordination entre la croissance du charançon du pois et celle de son hôte végétal: «un transfert de signification [Bedeutungsübertragung] a donc dû s'effectuer depuis l'archétype [Urbild] du pois à l'archétype du charançon du pois, et a permis que prévale entre eux l'harmonie » (Uexküll \& Kriszat 1956 : 122). Uexküll ne croit ni à un apprentissage du charançon par essais successifs, ni à une sélection darwinienne, mais à une perception originelle par le charançon d'une concordance entre le pois et ses possibilités d'action.

Le darwinisme a vaincu et ôté sa légitimité à ce type de discours. En outre, les mécanismes rétroactifs et auto-correcteurs darwino-compatibles, issus du concept de réflexe, ont depuis servi à neutraliser les discours téléologiques (principalement après l'avènement de la cybernétique), ou à réduire des comportements complexes à des hiérarchies d'opérations en feedback (Miller, Galanter \& Pribram 1960). Autrement dit, les déclarations d'Uexküll sur les rencontres harmonieuses entre archétypes peuvent être confortablement ignorées, sans pour autant abandonner l'emploi d'un métalangage sémiotique.

Sebeok a en quelque sorte dégermanisé partiellement Uexküll en effaçant la composante vitaliste et les élans qui lui venaient de la Naturphilosophie. Surtout, il l'a intégré à une description œcuménique des signes dont l'une des sources essentielles est Peirce, évidemment mieux connu du monde anglophone. À Peirce il emprunte la trichotomie icône/indice/symbole (non sans lui ajouter le symptôme, le signal et le nom, d'une manière bien peu peircienne) et il lui assigne une place privilégiée dans l'histoire : celle du premier penseur moderne qui, avec Saussure, aurait circonscrit le domaine de la sémiotique et l'aurait enraciné dans la sensorialité, l'affectivité et la cognition ; et celle du penseur qui aurait prolongé la préoccupation lockienne pour le rôle cognitif et épistémique des signes (Sebeok 2001 [1994] : 5). Toutes affirmations dont on pourrait discuter longuement, mais qu'on se contentera de rapporter ici.

Sebeok est sûrement plus proche de Uexküll quand il prend la pose kantienne de l'inaccessibilité de la chose en soi pour l'organisme plongé dans son monde subjectif : "Whether there is a reality behind signs - perhaps what Heraclitus called logos, the repeatable structure that secures for any object its ideal unity and stability, [...] humanity can never be sure » (ibid. : 34). Quant à l'extension de l'Umwelt à l'environnement humain, dont le langage fait partie, elle s'opère en empruntant à l'école de Moscou-Tartu la notion de système de modélisation du monde : les langues sont prioritairement des systèmes de catégorisation du monde destinés au guidage des individus, secondairement des moyens de communication. La perspective est proche de Gipper.

\section{EN GUISE DE CONCLUSION}

Pour clore cette tentative de contextualisation des thèmes abordés ici, nous nous contenterons de quelques mots sur leur nouvelle actualité. Nous avons déjà vu que la biosémiotique était un programme de recherche actif. Quant à la linguistique psychologique, elle a pris aujourd'hui le nom de linguistique cogni- 
tive. Quoiqu'issue d'un milieu intellectuel bien différent des variétés élaborées par les philosophes, psychologues et linguistes de langue allemande, elle n'en présente pas moins des parallèles avec ces dernières.

Les deux courants ont des propensions empiristes, tant en ce qui regarde la sémantique que la formation des structures linguistiques, d'où l'importance accordée à l'usage et à la fréquence chez Langacker et Bybee comme chez Hermann Paul (Fortis 2018b). Le parallélisme entre formes et représentations qu'on trouve chez Langacker n'est pas sans rappeler Wundt ; en témoignerait leur traitement des impersonnels. De même, la caractérisation du sujet comme dominierende Vorstellung a sa contrepartie chez Talmy et Langacker, qui emploient la figure gestaltiste pour gloser cognitivement cette notion grammaticale. Le rôle considérable de l'attention chez Talmy évoque par son ubiquité le concept d'aperception. Enfin, les deux courants sont centrés sur l'individu et ses représentations et tendent à minorer les facteurs pragmatiques (avec les réserves évoquées plus haut pour le domaine germanique).

Ces similitudes coexistent avec des différences marquées : la psychologie des linguistes cognitivistes est " spontanée », construite avec des éléments empiristes et gestaltistes, mais elle n'est pas appuyée sur un modèle particulier, autrefois herbartien ${ }^{22}$. Les spéculations sur l'origine du langage ou le rôle du sens étymologique dans la constitution de la forme interne font place désormais à une perspective synchronique, du moins dans la linguistique cognitive des débuts, avant qu'elle ne se connecte à la linguistique fondée sur l'usage. Nous ne nous plaindrons pas de l'effacement des jugements de valeur sur les langues non indoeuropéennes qu'on trouve chez Steinthal et Wundt. Les typologues cognitivistes sont des relativistes neutres à cet égard ${ }^{23}$.

Peut-être parce qu'ils ont moins la tête philosophique que leurs prédécesseurs germaniques, et ont été nourris au générativisme, les linguistes cognitivistes ne se posent pas la question du statut de la langue, ni du rapport entre l'unité de signification et la variabilité des contenus psychiques. La distinction entre les « concepts » et les signifiés linguistiques est thématisée dans les deux courants : l'imagerie conventionnelle de Langacker est une sorte d'équivalent de la forme interne. Néanmoins cette distinction gagnerait à être clarifiée dans la linguistique cognitive. De même, la théorie des métaphores conceptuelles aurait avantage, pour plus d'acuité épistémologique, à redécouvrir la notion de forme interne prônée par Marty. Une confrontation des deux époques pourrait donc être fructueuse. Espérons que la lecture du présent dossier, en plus d'éclairer le lecteur, lui suggérera encore d'autres pistes de réflexion.

22 À l'exception de la théorie du prototype, importée de la psychologie sous une forme simplifiée (Fortis 2018a).

23 Relativistes en ce qui concerne les structures linguistiques, analysées généralement comme tout à fait spécifiques à une langue donnée, mais universalistes en ce qui regarde les opérations cognitives ou les fonctions générales prototypiquement remplies par les catégories et structures grammaticales. Croft (2001) illustre bien cette double perspective. Des éléments universalistes se trouvent chez Talmy (2000 [1985]), qui postule une structure universelle de l'énoncé. L'influence de l'universalisme chomskyen est perceptible chez cet ex-sémanticien générativiste. 


\section{BIBLIOGRAPHIE}

Ach, Narziß. 1905. Über die Willenstätigkeit und das Denken. Göttingen : Vandenhoeck \& Ruprecht.

d'Alembert, Jean Le Rond. 1751. Discours préliminaire des Éditeurs. Encyclopédie, ou Dictionnaire raisonné des sciences, des arts et des métiers, dir. par Denis Diderot \& Jean Le Rond d'Alembert. Paris : Briasson. I-XLV.

Araujo, Saulo de Freitas. 2016. Wundt and the Philosophical Foundations of Psychology. A Reappraisal. Cham (Suisse) : Springer.

Barasch, Moshe. 1990. Modern Theories of Art 1. From Winckelmann to Baudelaire. New York \& Londres : New York University Press.

Becker, Karl Ferdinand. 1841 [1827]. Organism der Sprache. Francfort-sur-le-Main : Kettembeil.

Becker, Karl Ferdinand. 1843. Ausführliche Deutsche Grammatik als Kommentar der Schulgrammatik, vol. 2. Francfort-sur-le-Main : Verlag von S. F. Kettembeil.

Beer, Theodor, Albrecht Bethe \& Jakob von Uexküll. 1899. Vorschläge zu einer objektivierenden Nomenklatur in der Physiologie des Nervensystems. Biologisches Centralblatt 19 : 517-521.

Beiser, Frederick C. 2014. The Genesis of Neo-Kantianism. Oxford: Oxford University Press.

Binet, Alfred. 1903. L'étude expérimentale de l'intelligence. Paris : Schleicher Frères.

Braunstein, Jean-François. 2012. L'invention française du "psychologisme» en 1828. Revue d'histoire des sciences $65: 197-212$.

Bréal, Michel. 1868. Les idées latentes du langage. Leçon faite au Collège de France pour la réouverture du cours de grammaire comparée le 7 décembre 1868. Paris : Librairie de L. Hachette et $\mathrm{C}^{\mathrm{ie}}$.

Brentari, Carlo. 2015. Jakob von Uexküll. The Discovery of the Umwelt between Biosemiotics and Theoretical Biology. Dordrecht : Springer.

Burkard, Thorsten. 2003. Die lateinische Grammatik im 18. und frühen 19. Jahrhundert. Von einer Wortarten- zu einer Satzgliedgrammatik. Ellipsentheorie, Kasuslehre, Satzglieder. Germania latina - Latinitas teutonica. Politik, Wissenschaft, humanistische Kultur vom späten Mittelalter bis in unsere Zeit, dir. par Eckhard Keßler \& Heinrich C. Kuhn. Munich : Wilhelm Fink. 781-830.

Carroy, Jacqueline, Annick Ohayon \& Régine Plas. 2006. Histoire de la psychologie en France, $X I X^{e}-X X^{e}$ siècles. Paris : La Découverte.

Claparède, Édouard. 1902. Revue générale sur les principes de la psychologie animale (Loeb, Nagel, von Uexküll, Norman, Bethe, Bechterew, Edinger, Wasmann, Jennings, Arnhart, Ziegler, etc.). L'Année psychologique 9 : 483-494.

Croft, William. 2001. Radical Construction Grammar: Syntactic Theory in Typological Perspective. Oxford: Oxford University Press.

Delacroix, Henri. 1924. Le langage et la pensée. Paris : Librairie Félix Alcan.

Driesch, Hans. 1922. Geschichte des Vitalismus. Leipzig : Johann Ambrosius Barth.

Dugas, Ludovic. 1895. Recherches expérimentales sur les différents types d'images. Revue philosophique de la France et de l'étranger 39 : 285-292. 
Elffers-van Ketel, Els. 1991. The Historiography of Grammatical Concepts. 19th and 20th-Century Changes in the Subject-Predicate Conception and the Problem of their Historical Reconstruction. Amsterdam \& Atlanta : Rodopi.

Elffers, Els. 1999. Psychological linguistics. Geschichte der Sprachtheorie 4. Sprachtheorien der Neuzeit, dir. par Peter Schmitter. Tübingen : Narr. 301-341.

Favareau, Donald. 2010. Essential Readings in Biosemiotics. Anthology and Commentary. Dordrecht : Springer.

Feuerhahn, Wolf. 2002. Comment la psychologie empirique est-elle née ? Archives de Philosophie 65 : 47-64.

Feuerhahn, Wolf. 2009. Du milieu à l'Umwelt: enjeux d'un changement terminologique. Revue philosophique de la France et de l'étranger 134 : 419-438.

Fisette, Denis. 2015. La théorie des signes locaux de Hermann Lotze et la controverse empirisme-nativisme au $\mathrm{XIX}^{\mathrm{e}}$ siècle. Lotze et son héritage, dir. par Federico Boccaccini. Bruxelles, Berne \& Berlin : Peter Lang. 45-71.

Formigari, Lia. 1994. La sémiotique empiriste face au kantisme. Liège : Mardaga.

Fortis, Jean-Michel. 2015. Sapir et le sentiment de la forme. Histoire Épistémologie Langage 37(2) : 153-174.

Fortis, Jean-Michel. 2018a. Prototype Theory: On the Origins of a Theoretical Patchwork and its Transfer to Linguistics. Theorization and Representations in Linguistics, dir. par Viviane Arigne \& Christiane Rocq-Migette. Newcastle upon Tyne : Cambridge Scholars Publishing. 168-216.

Fortis, Jean-Michel. 2018b. Le changement linguistique selon Joan Bybee, ou Hermann Paul redivivus. Histoire des langues et histoire des représentations linguistiques, dir. par Bernard Colombat, Bernard Combettes, Valérie Raby \& Gilles Siouffi. Paris : Honoré Champion. 199-218.

Fortis, Jean-Michel. 2018c. Anderson's Case Grammar and the History of Localism. Substance-based Grammar. The (Ongoing) Work of John Anderson, dir. par Roger Böhm \& Harry van der Hulst. Amsterdam : John Benjamins. 113-198.

Fortis, Jean-Michel. 2019. On Sapir's Notion of Form/Pattern and its Aesthetic Background. Form and Formalism in Linguistics, dir. par James McElvenny. Berlin : Language Science Press. 59-88.

Freuler, Léon. 1997. La crise de la philosophie au XIXe siècle. Paris : Vrin.

Gardiner, Alan H. 1932. The Theory of Speech and Language. Oxford : Clarendon Press.

Gipper, Helmut. 1963. Bausteine zur Sprachinhaltsforschung. Neuere Sprachbetrachtung im Austausch mit Geistes- und Naturwissenschaft. Düsseldorf: Pädagogischer Verlag Schwann.

Goclenius, Rudolph. 1590. ЧҮХОАОГІА: hoc est, de hominis perfectione, animo, et in primis ortu hujus, commentationes ac disputationes quorundam Theologorum \& Philosophorum nostrae aetatis. Marburg: Ex officina typographica Pauli Egenolphi.

Gomperz, Heinrich. 1908. Weltanschauungslehre: Ein Versuch die Hauptprobleme der allgemeinen theoretischen Philosophie geschichtlich zu entwickeln und sachlich zu bearbeiten, vol. 2 : Noologie, partie 1: Einleitung und Semasiologie. Iéna \& Leipzig : Diederichs. 
Graffi, Giorgio. 2001. 200 Years of Syntax: A Critical Survey. Amsterdam \& Philadelphie : John Benjamins.

Grot, Nicolas von. 1895. Die Begriffe der Seele und der psychischen Energie in der Psychologie. Archiv für systematische Philosophie IV(3) : 257-335.

Harrington, Anne. 1996. Reechanted Science. Holism in German Culture from Wilhelm II to Hitler. Princeton : Princeton University Press.

Hatfield, Gary. 1990. The Natural and the Normative. Cambridge (MA) : MIT Press.

Helmholtz, Hermann von. 1867. Handbuch der physiologischen Optik, 3 vol. Leipzig : L. Voss.

Herbart, Johann Friedrich. 2005. Les points principaux de la métaphysique, introduit, traduit et annoté par Carole Maigné. Paris : Vrin.

Herder, Johann Gottfried. 1978 [1772]. Abhandlung über den Ursprung der Sprache, éd. par Wolfgang Proß. Munich : Hanser.

Herder, Johann Gottfried. 1799. Metakritik. Leipzig : J. F. Hartknoch.

Hockett, Charles F. 1958. A Course in Modern Linguistics. New York: The MacMillan Company.

Humboldt, Wilhelm von. 1836. Über die Kawi-Sprache auf der Insel Java nebst einer Einleitung über die Verschiedenheit des menschlichen Sprachbaues und ihren Einflu $\beta$ auf die geistige Entwicklung des Menschengeschlechts. Berlin: Königliche Akademie der Wissenschaften.

Humboldt, Wilhelm von. 1974 [1836]. Introduction à l'œuvre sur le kavi et autres essais. Traduction, introduction et notes de Pierre Caussat. Paris : Seuil.

Hume, David. 1978 [1739-1740]. A Treatise of Human Nature: Being an Attempt to Introduce the Experimental Method of Reasoning into Moral Subjects, éd. par L. A. Selby-Bigge, rév. de P. H. Nidditch. Oxford : Clarendon Press.

Humphrey, George. 1951. Thinking: An Introduction to its Experimental Psychology. Londres \& New York : Methuen \& Wiley.

Husserl, Edmund. 1959 [1900]. Recherches logiques, tome I : Prolégomènes à la logique pure. Trad. par H. Elie, A. L. Kelkel \& R. Schérer. Paris: Presses universitaires de France [publication originale : Logische Untersuchungen. Erster Theil: Prolegomena zur reinen Logik. Leipzig : Von Veit \& Comp. 1900].

James, William. 1890. The Principles of Psychology, vol. 1. New York : Holt.

Kant, Emmanuel. 1985 [1786]. Premiers principes métaphysiques de la science de la nature. Euvres philosophiques II. Paris : Gallimard. 363-494.

Knobloch, Clemens. 1988. Geschichte der Psychologische Sprachauffassung in Deutschland von 1850 bis 1920. Tübingen : Max Niemeyer Verlag.

Kuehn, Manfred. 1987. Scottish Common Sense in Germany, 1768-1800. A Contribution to the History of Critical Philosophy. Kingston \& Montréal : McGillQueen's University Press.

Kull, Kalevi. 2001. Jacob von Uexküll: An Introduction. Semiotica 134(1/4) : 1-59.

Kusch, Martin. 1995. Psychologism. A Case Study in the Sociology of Philosophical Knowledge. Londres \& New York : Routledge.

Lipps, Theodor. 1897. Raumästhetik und geometrisch-optische Täuschungen. Leipzig : Barth. 
Lipps, Theodor. 1907. Ästhetik. Systematische Philosophie, dir. par Wilhelm Dilthey. Berlin \& Leipzig : B. G. Teuber. 349-387.

Lotze, Hermann. 1852. Medicinische Psychologie oder Physiologie der Seele. Leipzig : Weidmann'sche Buchhandlung.

Madvig, Johan Nikolai. 1875 [1856]. Vom Entstehen und Wesen der grammatischen Bezeichnungen. Kleine Philologische Schriften. Leipzig : B. G. Teubner. 98-290.

Maigné, Carole. 2005. Le réalisme rigoureux de J. F. Herbart. Les points principaux de la métaphysique, par J. F. Herbart, introduit, traduit et annoté par Carole Maigné. Paris : Vrin. 7-162.

Marty, Anton. 1910. Die „logische”, „localistische” und andere Kasustheorien. Halle-sur-Saale : Verlag von Max Niemeyer.

Marty, Anton. 2017 [1875]. Sur l'origine du langage. Trad. par Marc de Launay. Paris: Hermann [publication originale: Über den Ursprung der Sprache. Würzburg : A. Stuber's Buchhandlung].

Mill, John Stuart. 1843. A System of Logic, Ratiocinative and Inductive, Being a Connected View of the Principles of Evidence, and the Methods of Scientific Investigation, vol. II. Londres : John W. Parker.

Miller, George A., Eugene Galanter \& Karl H. Pribram. 1960. Plans and the Structure of Behavior. New York : Holt, Rinehart \& Winston.

Morel, Charlotte, éd. 2017. L'Allemagne et la querelle du matérialisme (1848-1866). Une crise oubliée ? Paris : Garnier.

Müller, Johannes. 1834-1840. Handbuch der Physiologie des Menschen für Vorlesungen. Coblence : J. Hölscher.

Müller, Max. 1873. Lectures on Mr. Darwin's Philosophy of Language. Fraser's Magazine VII(XLI) : 525-678.

Nicolas, Serge. 2002. La fondation de la psychophysique de Fechner: des présupposés métaphysiques aux écrits scientifiques de Weber. L'Année psychologique 102(2) : 255-298.

Noiré, Ludwig. 1877. Der Ursprung der Sprache. Mayence : Victor von Zabern.

Nuchelmans, Gabriel. 1986. The Historical Background to Locke's Account of Particles. Logique et Analyse 113 : 53-71.

Paul, Hermann. 1920 [1880]. Prinzipien der Sprachgeschichte. Halle-sur-Saale : Verlag von Max Niemeyer.

Percival, Keith W. 1976. On the Historical Source of Immediate Constituent Analysis. Notes from the Linguistic Underground, dir. par James D. McCawley. New York : Academic Press (Syntax and Semantics, 7). 229-242.

Raby, Valérie. 2018. Les théories de l'énoncé dans la grammaire générale. Lyon: ENS Éditions.

Reid, Thomas. 1997 [1764]. An Inquiry into the Human Mind on the Principles of Common Sense, éd. par Derek R. Brookes. University Park : Pennsylvania State University Press.

Ribot, Théodule. 1875. La psychologie anglaise contemporaine. Paris: Germer Baillière.

Ribot, Théodule. 1919. L'évolution des idées générales. Paris : Librairie Félix Alcan. 
Ribot, Théodule. 2003 [1879]. La psychologie allemande contemporaine. Introduction de Serge Nicolas. Paris : L'Harmattan.

Ricken, Ulrich. 1969. La liaison des idées selon Condillac et la clarté du français. Dixhuitième siècle 1 : 179-193.

Romand, David. 2010. «Das Körper-Seele Problem ». La question du rapport du psychique au physique dans la psychologie allemande du XIX ${ }^{\mathrm{e}}$ siècle. Revue de synthèse 131(1) : 35-51.

Romand, David. 2015. Theodor Waitz's Theory of Feelings and the Rise of Affective Sciences in the Mid-19th Century. History of Psychology 18(4) : 385-400.

Romand, David. 2018. An Unorthodox Herbartian in Graz: Joseph Wilhelm Nahlowsky's “Affective Herbartianism”. Herbart als Universitätslehrer, dir. par Jean-François Goubet \& Rainer Bolle. Iéna : Garamond Verlag. 203-220.

Romanes, George John. 1883. Mental Evolution in Animals. Londres : Kegan Paul, Trench \& Co.

Rorty, Richard. 1990 [1979]. L’homme spéculaire. Paris : Seuil. Trad. par Thierry Marchaisse [publication originale: Philosophy and the Mirror of Nature. Princeton, NJ : Princeton University Press. 1979].

Samain, Didier. 2014. Portrait du linguiste en jeune grammairien. Recherches sémiotiques / Semiotic Inquiry 34(1-2-3) : 137-156.

Scheler, Max. 1928. Die Stellung des Menschen im Kosmos. Darmstadt : Otto Reichl.

Sebeok, Thomas A. 1963. Review of Communication Among Social Bees by Martin Lindauer; Porpoises and Sonar by Winthrop N. Kellogg; Man and Dolphin by John C. Lilly. Language 39(3) : 448-466.

Sebeok, Thomas A. 1979. The Sign and its Masters. Austin \& Londres: The University of Texas Press.

Sebeok, Thomas A. 2001 [1994]. Signs: An Introduction to Semiotics. Toronto : University of Toronto Press.

Seuren, Pieter. 1998. Western Linguistics. An Historical Introduction. Oxford \& Malden : Blackwell Publishing.

Simonato, Elena. 2005. Une linguistique énergétique en Russie au seuil du XXe siècle. Essai d'analyse épistémologique. Berne : Peter Lang.

Spiess, Gustav Adolf. 1844. Physiologie des Nervensystems. Braunschweig : Vieweg.

Steinthal, Heymann. 1855. Grammatik, Logik und Psychologie. Ihre Principien und ihr Verhältniss zu einander. Berlin : F. Dümmler.

Steinthal, Heymann. 1871. Einleitung in die Psychologie und Sprachwissenschaft. Berlin : F. Dümmler.

Talmy, Leonard. 2000 [1985]. Lexicalization Patterns. Toward a Cognitive Semantics, par L. Talmy, vol. 2. Cambridge, Mass. : MIT Press. 21-146 [publication originale : Lexicalization Patterns: Semantic Structure in Lexical Form. Language Typology and Syntactic Description, vol. 3, dir. par T. Shopen. Cambridge : Cambridge University Press. 1985. 57-149].

Uexküll, Jakob von. 1902. Im Kampf um die Tierseele. Wiesbaden : J. F. Bergmann.

Uexküll, Jakob von \& Georg Kriszat. 1956. Streifzüge durch die Umwelten von Tieren und Menschen. Bedeutungslehre. Hambourg : Rowohlt. 
van Ginneken, Jac. 1907. Principes de linguistique psychologique. Essai de synthèse. Amsterdam, Paris \& Leipzig : Van der Vecht, Rivière \& Harrassowitz.

Vidal, Fernando. 2011. The Sciences of the Soul. The Early Modern Origins of Psychology. Trad. par Saskia Brown. Chicago \& Londres: The University of Chicago Press [publication originale: Les sciences de l'âme. XVI -XVIII siècles. Paris : Honoré Champion. 2006].

Vischer, Robert. 1873. Über das Optische Formgefühl. Ein Beitrag zur Aesthetik. Leipzig : Hermann Credner.

Waitz, Theodor. 1849. Lehrbuch der Psychologie als Naturwissenschaft. Brauschweig: Vieweg.

Weil, Henri. 1844. De l'ordre des mots dans les langues anciennes comparées aux langues modernes. Paris : Joubert.

Winckelmann, Johann. 1764. Geschichte der Kunst des Alterthums, vol. 1. Dresde : Waltherische Hof-Buchhandlung.

Wundt, Wilhelm. 1863. Vorlesungen über die Menschen- und Thierseele. Leipzig : Leopold Voß.

Wundt, Wilhelm. 1880. Logik. Eine Untersuchung der Prinzipien der Erkenntnis und der Methoden wissenschaftlicher Forschung. Stuttgart : Enke.

Wundt, Wilhelm. 1912a. Elemente der Völkerpsychologie: Grundlinien einer psychologischen Entwicklungsgeschichte der Menschheit. Leipzig : A. Kröner.

Wundt, Wilhelm. 1912b. Völkerpsychologie. Eine Untersuchung der Entwicklungsgesetze von Sprache, Mythus und Sitte, vol. 2, partie 2. Leipzig: Verlag von Wilhelm Engelmann.

Wundt, Wilhelm. 1920. Erlebtes und Erkanntes. Stuttgart : A. Kröner. 
